\title{
Quantitative ET in Materials Chemistry
}

\author{
Heiner Friedrich ${ }^{1,2}$
}

1. Laboratory of Materials and Interface Chemistry and Center of Multiscale Electron Microscopy, Department of Chemical Engineering and Chemistry, Eindhoven University of Technology, Eindhoven, The Netherlands.

2. Institute for Complex Molecular Systems, Eindhoven University of Technology, Eindhoven, The Netherlands.

The controlled segregation of primary building blocks (atoms, molecules, colloids) into different phases, delineated by interfaces, is a general process in materials synthesis and employed in many fields including colloid chemistry, heterogeneous catalysis, and functional molecular systems [1]. To rationally design complex soft materials, we must know their morphological evolution over all relevant length and time scales. In our work we frequently combine (time-resolved) sample preparation with (cryo) (S)TEM electron tomography and quantification of structures by image analysis to facilitate this task [1-4]. Here, our latest results on quantitative ET of porous silica particles [5] and conductive carbon nanotube compacts [6] are presented.

Micrometer sized porous silica particles which are used in high performance liquid chromatography (Kromasil) were imaged in 3D by low convergence-angle annular dark-field STEM (LC-ADF-STEM) tomography (Figure 1a,b). The reconstruction was segmented, as shown in Figure 1c,d, using a local volume threshold corresponding to the average particle porosity as determined by $\mathrm{N}_{2}$ gas adsorption (65.3 vol\% pores). This enabled us to describe the pore network over two orders of magnitude (10 nm pores in $2 \mu \mathrm{m}$ sphere). The ET derived pore size distribution (PSD) from two different regions within the particle (center of the particle and edge region) matched very well with the PSD derived from $\mathrm{N}_{2}$ physisorption (Figure 1e). Pores of between 8 and $16 \mathrm{~nm}$ were found which were homogeneously distributed throughout the particle. Such quantitative description of the pore network in terms of interconnectivity, homogeneity and pore width significantly aids our ongoing studies to control silica gelation into structures with a tuneable/graded porosity suitable for separation of large molecules [5].

Gaining insight into the fundamental packing rules of rod like colloids (e.g. in dependence of their aspect ratio) is critical to developing a bottom-up understanding of their structural and functional properties. To this end we employed polydisperse populations (length and width) of carbon nanotubes (CNT) of varying average aspect ratio which were assembled into randomly packed porous compacts by vacuum filtration. LC-ADF-STEM ET was carried out on microtomed sections of up to $1 \mu \mathrm{m}$ in thickness. Segmentation was carried out by template matching using cylinder templates of varying inner and outer diameter to account for the polydispersity in the sample. The formed networks (Figure 2a) were quantified in terms of packing density (Figure 2b), contact number and area, and CNT segment length between contacts. For our nanoscale colloidal rod system an excellent match with the macroscopic random contact model (Figure 2b, red line) was found in terms of packing density and contact number per rod. From the network topology and applying Kirchhoff's circuit laws a bottom upmodelling of the conductive properties of the CNT network now becomes feasible [6].

Above examples illustrate the importance of Quantitative ET in Materials Chemistry to resolve the multiscale morphology of complex soft matter enabling the tuning of their functional properties [7]. 
References:

[1] J. Patterson et al, Acc. Chem. Res. 50 (2017), p.1495.

[2] B. McKenzie et al, Angew. Chem. Int. Ed. 54 (2015), p.2457.

[3] M. Wirix et al, Nanoletters 14 (2014), p. 2033.

[4] T. Eggenhuisen et al, Chem. Mater. 25 (2013), p.890.

[5] A. Fijneman et al, in preparation.

[6] K. Gnanasekaran, G. de With and H. Friedrich in preparation.

[7] HF would like to thank K. Gnanasekaran, A. Fijneman, A. Keizer, G. de With and N. Sommerdijk. This work has been funded in parts by the European Commission FP7-ITN Manano (264710), H2020ITN Multimat (676045) and the Dutch Polymer Institute (EU-FP-002).
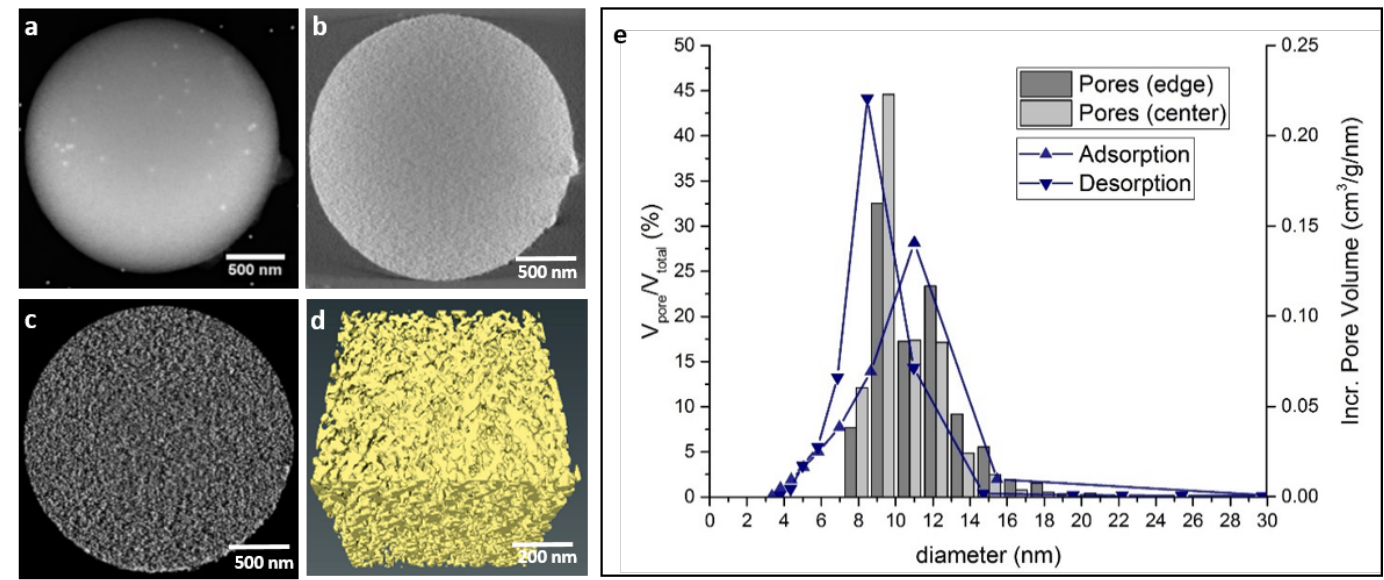

Figure 1. (a) STEM image of a Kromasil 2/10 particle. (b) central-slice of reconstruction (c) centralslice after segmentation. (d) Representative 3D volume rendering of the pore structure. (e) Pore size distribution as determined from ET and $\mathrm{N}_{2}$ gas adsorption (line).

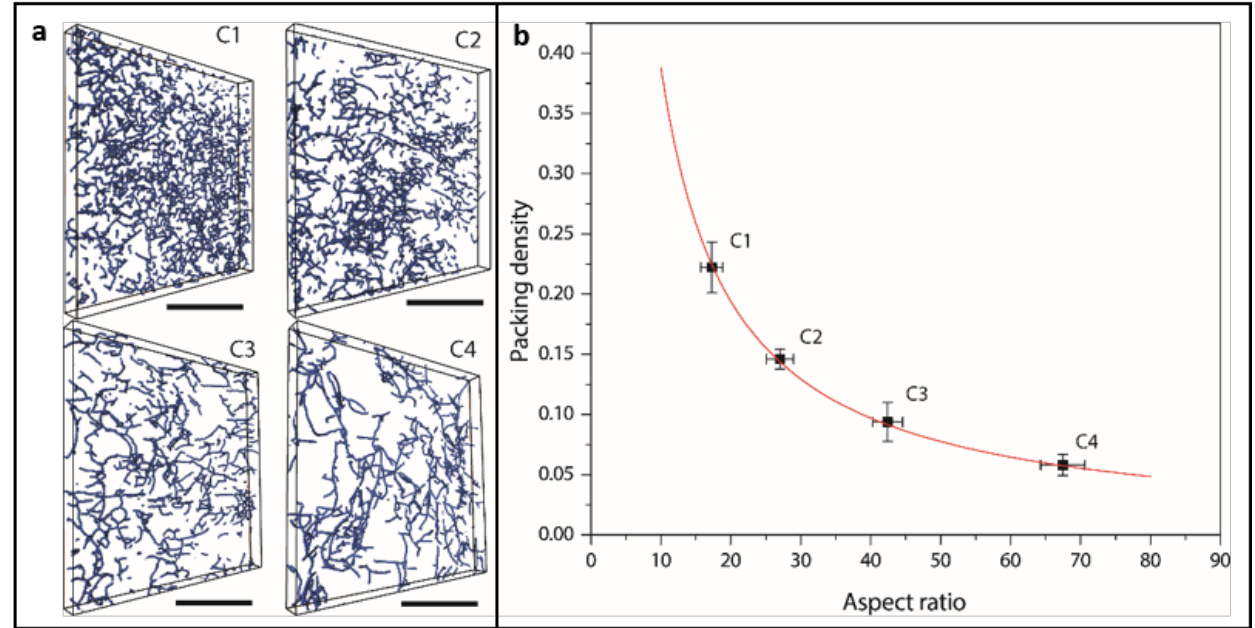

Figure 2. (a) volume rendering of CNT networks formed by varying the aspect ratio of the nanorods. Scale bars are $500 \mathrm{~nm}$. (b) packing density (black squares) plotted as a function of the average aspect ratio with fit to macroscopic random contact model (red line). 